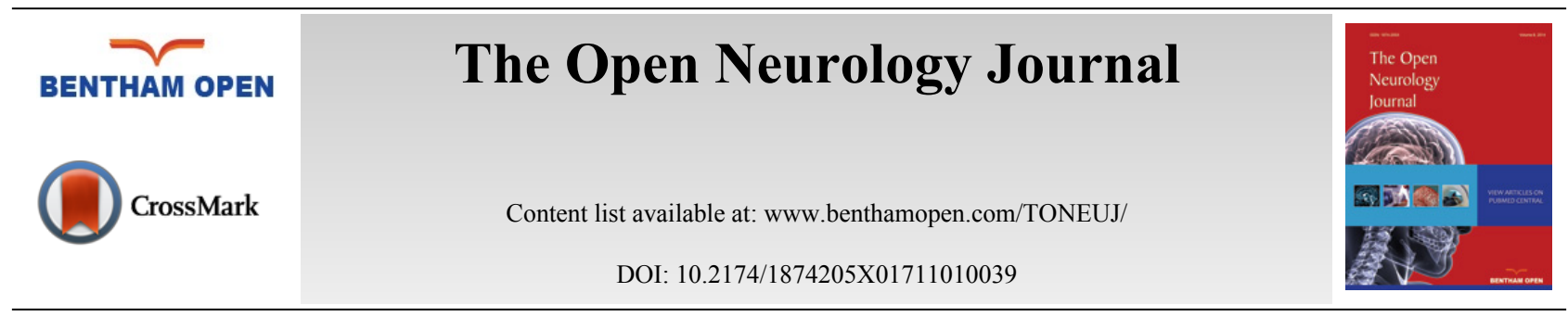

RESEARCH ARTICLE

\title{
Robot-Embodied Neuronal Networks as an Interactive Model of Learning
}

\author{
Abraham M Shultz ${ }^{1}$, Sangmook Lee ${ }^{2}$, Mary Guaraldi ${ }^{2}$, Thomas B. Shea ${ }^{2, *}$ and Holly A. Yanco ${ }^{1}$ \\ ${ }^{I}$ Robotics Laboratory, Department of Computer Science, USA \\ ${ }^{2}$ Laboratory for Neuroscience, Department of Biological Sciences University of Massachusetts Lowell, Lowell, MA \\ 01854, USA
}

Received: February 20, 2017

Revised: June 19, 2017

Accepted: August 03, 2017

\begin{abstract}
:
Background and Objective:

The reductionist approach of neuronal cell culture has been useful for analyses of synaptic signaling. Murine cortical neurons in culture spontaneously form an ex vivo network capable of transmitting complex signals, and have been useful for analyses of several fundamental aspects of neuronal development hitherto difficult to clarify in situ. However, these networks lack the ability to receive and respond to sensory input from the environment as do neurons in vivo. Establishment of these networks in culture chambers containing multi-electrode arrays allows recording of synaptic activity as well as stimulation.
\end{abstract}

\section{Method:}

This article describes the embodiment of ex vivo neuronal networks neurons in a closed-loop cybernetic system, consisting of digitized video signals as sensory input and a robot arm as motor output.

\section{Results:}

In this system, the neuronal network essentially functions as a simple central nervous system. This embodied network displays the ability to track a target in a naturalistic environment. These findings underscore that ex vivo neuronal networks can respond to sensory input and direct motor output.

\section{Conclusion:}

These analyses may contribute to optimization of neuronal-computer interfaces for perceptive and locomotive prosthetic applications. Ex vivo networks display critical alterations in signal patterns following treatment with subcytotoxic concentrations of amyloid-beta. Future studies including comparison of tracking accuracy of embodied networks prepared from mice harboring key mutations with those from normal mice, accompanied with exposure to Abeta and/or other neurotoxins, may provide a useful model system for monitoring subtle impairment of neuronal function as well as normal and abnormal development.

Keywords: Multi-electrode array, Neuronal network, Cortical neuronal culture, Learning, Plasticity, Sensory input, Cybernetics, Manus robot arm.

\section{INTRODUCTION}

The advent of culture dishes within which is embedded an array of electrodes has allowed interfacing of cultured neurons with computer software for recording and stimulation. This robust approach has been utilized to gain major advances in understanding of synaptogenesis and neuronal signaling [1-6], as well as gaining insight into factors that promote these processes and underlie or accelerate neurodegeneration [7 -9]. These Multi-Electrode Arrays

\footnotetext{
"Address correspondence to this author at the Laboratory for Neuroscience, Department of Biological Sciences University of Massachusetts Lowell, Lowell, MA 01854, USA, Tel: 978-934-2881; Fax: 978-934-3044; E-mail: thomas_shea@uml.edu
} 
(MEA) and the appropriate software have demonstrated that cultured neurons spontaneously undergo synaptogenesis and establish a functional network capable of transmitting information across multiple neurons. Over the course of approximately 1 month, the spontaneous signal patterns generated by these ex vivo neuronal networks undergo developmental conversion from individual "spikes" to complex bursts that consist of multiple signals [3 - 6, 10 - 15]. This conversion recapitulates the delayed establishment of inhibitory neuronal activity observed in situ [16 - 21].

External stimulation hastens the establishment of a signaling pattern characteristic of mature cultures [2, 3, 12, 22 24], confirming that ex vivo networks are capable of responding to external stimulation. Our studies in which networks were subjected to multiple stimulation regimens using a digitized synaptic signal further demonstrated that ex vivo networks altered their signaling patterns in a manner analogous to long-term potentiation in situ [6].

A more robust and "real-world" approach towards activity and responsiveness of ex vivo neuronal networks has been to interface them with robotics $[25,26]$. Herein, we provided ex vivo neuronal networks with sensory input using a digital video camera, and converting resultant network signaling to impulses capable of operating a robotic arm. The resultant "embodied" neuronal network therefore represents a simple central nervous system that processes incoming sensory input and generates corresponding motor output.

\section{MATERIALS and METHODS}

\subsection{Generation of Ex Vivo Neuronal Networks}

Dissociated cortical neurons from day 18 C57BL/6 mouse embryos were plated in B27-supplemented Neurobasal medium (Invitrogen, Carlsbad, CA) on poly-D-lysine/fibronectin-coated "MEA petri dishes" (Multichannel Systems, Reutlingen, Germany) containing 60 Titanium Nitride electrodes in an 8 by 8 grid arrangement; detailed information on generation of networks and of the MEAs themselves has been published previously [3 - 6, 27]. Sacrifice of pregnant females was carried out under procedures approved by our Institutional Animal Care and Use Committee.

\subsection{Recording of Network Activity}

Signaling was recorded using a MEA-1060-INV amplifier over 30 second intervals and collected via a DT9814 acquisition system and a software program ("Raptor") developed in our laboratory [3, 4] (available at https:/github.com/mtgjbird/Raptor). Raptor requires the LabView (National Instruments) run-time engine 2013 SP1 or later http://www.ni.com/download/labview-run-time-engine-2013-sp1/4540/en/. Other appropriate software is available $[1,28]$. Networks were stimulated using a $1 \mathrm{sec}$ digitized synaptic signal $1 \mathrm{mV}$ in amplitude as in our prior studies [3, 4, 27]. Output was graphed and analyzed in Excel.

After culturing for 1 month to allow maturation of networks (confirmed by development of spontaneous signal streams rich in complex bursts [27], networks were segmented into two equivalent halves by slicing across the petri dish with a scalpel. This segmentation was not essential, but served to generate two independent "hemispheres." Separation was confirmed by phase-contrast microscopy Fig. (1) and by confinement of responding signals following stimulation within the half containing the electrode to which the exogenous signal was delivered (not shown). Networks were utilized immediately after segmentation, and therefore there was not sufficient time for regeneration of connections across the slice.

\subsection{Interfacing of Ex Vivo Neuronal Networks with Video Input and Robotic Output}

The complete software package, developed in our laboratory, and used for real-time interfacing our ex vivo neuronal networks with digital video input and robotic output is available at https:/github.com/ab3nd/ NeuronRobotInterface.

\subsection{Conversion of Video Signals and Stimulation of Neuronal Networks}

This was performed by the ROS module, "img_slicer," developed in our laboratory (available at https://github.com /ab3nd/NeuronRobotInterface/ tree/master/catkin_ws/src/img_slicer/src).

We utilized a solid red cup as a target object within a defined visual field. Notably, an object of any shape or hue could be utilized. Video was recorded with a tripod-mounted RGB digital camera. The resulting video was converted into HSV (Hue, Saturation, Value), and separated into hue, saturation, and value planes, which generated an accompanying image for each plane. This results in three images, one for each plane. The resultant hue image was subjected to thresholding to convert all pixels of the desired hue (red, in the example utilized herein) were converted to 
white, and all other pixels were converted to black (e.g., Fig. (2).
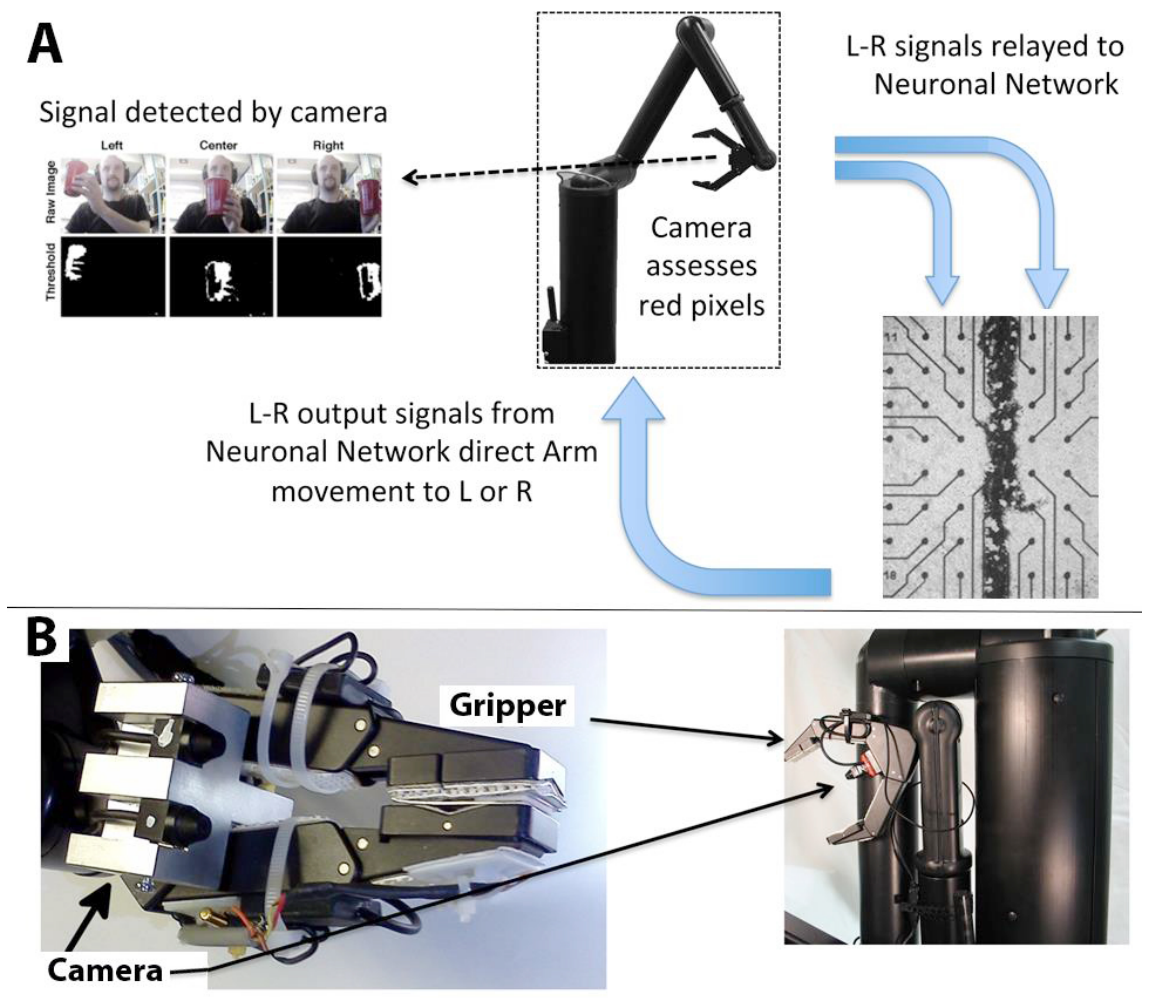

Fig. (1). Diagram of the embodied neuronal network.

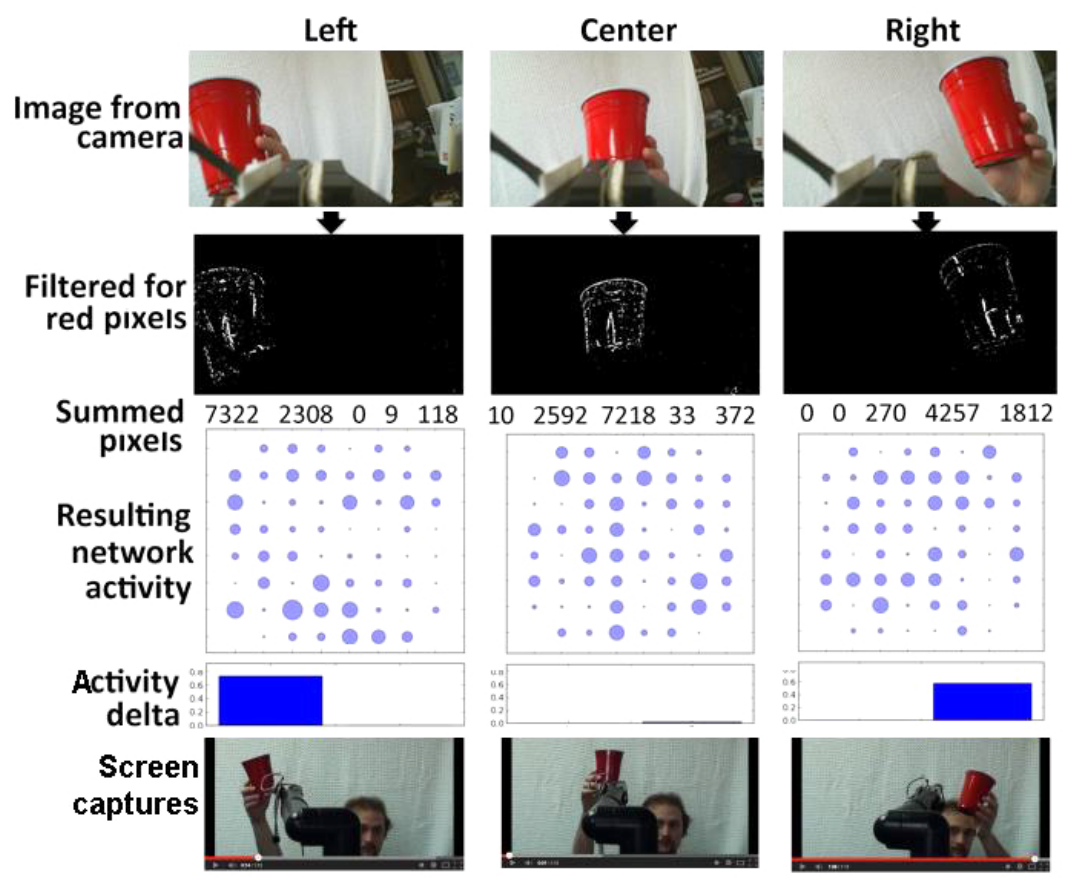

Fig. (2). A diagrammatic flow of information from the target to the network. Panels present the raw image of the target (a red cup in this example), the threshold image detected by the camera, the summation of pixels across the image ("Summed pixels"), a representative image of electrode activity as a result of image input ("Resulting network activity"), and a summation of total activity, which highlights the difference in activity within the left and right portions of the networks ("Activity delta"). Also presented are representative screen captures that demonstrate the robot arm tracking the target. 
To monitor movement of the target, the overall image field was separated into five equivalent vertical strips. The total white pixels in the leftmost 3 strips was then compared with the total in the rightmost 3 strips. If the sum of white pixels in the leftmost 3 strips was 500 more than the sum in the rightmost 3 strips, stimulation (via a digitized synaptic signal) [27] was delivered to the right of the network, in order to foster increased activity in the right portion of the network and vice versa. Accordingly, each difference of 500 pixels was translated into one synaptic signal. Delivery of synaptic signals to one half of the segmented network increased the activity of that segment Fig. (2); delivery of sufficient synaptic signals to increase the activity of that half of the network by a sufficient level prompted delivery of signals to the robot arm as described below.

\subsection{Conversion of Neuronal Network Activity to Motion Commands for the Manus Robotic Arm}

This was performed by the ROS module, "act_vector," developed in our laboratory (available at: https:/github.com/ ab3nd/NeuronRobotInterface/tree/master/ catkin_ws/src/activation_vector/src).

Each of the 60 channels of the MEA was recorded for $3 \mathrm{sec}$ intervals at a rate of $1000 \mathrm{samples} / \mathrm{sec}$. The mean and standard deviation of signaling on each channel was calculated, with one signal defined a $>3 \mathrm{x}$ the standard deviation of that channel.

A 60-element list (one for each channel) of activation values was initialized at zero. When a set of samples arrive, all the activation values $\mathrm{A}$ of each channel $\mathrm{n}$ were reduced according to the equation $A_{n}\left(t_{i}\right)=A_{n}\left(t_{i}-1\right) e^{-\beta(t i-t i-1)}$ as described by Hales et al. 2010. For each channel where a signal is detected, the activation value was incremented by 1 . Every 0.2 sec, the list of activation vectors was compared to the predefined "left" (L) and "right" (R) activation vectors by taking the 60 dimensional Euclidian distance between the current activation vector and the $\mathrm{L}$ and $\mathrm{R}$ vectors; these are fixed vectors reflecting increased activity on the $\mathrm{L}$ or $\mathrm{R}$ sides of the network. If the distance between the $\mathrm{L}$ or $\mathrm{R}$ activation vector approximates one of the fixed vectors (indicating that network activity was stronger on either the L or $\mathrm{R}$ side), a corresponding motion command was sent to the Manus robot arm, otherwise a stop command was sent to the Manus robot arm.

\section{RESULTS}

A diagram of information flow through the embodied neuronal network is presented in (Fig. 1).

Panel A describes basic information flow through the components of this system as described in Methods. The camera detects the position of the target, and transfers signals to the appropriate half of the segmented network. The stimulated network transmits motion commands to the arm, and the arm repositions in the direction of the target. This sequence repeats until the arm is situated directly in front of the target (if the target is stationary) or continues to track the target (if the target is moving). A portion of the segmented neuronal network is presented; black dots are the electrodes.

Panel B depicts the digital camera mounted above the grasping hand of the Manus robot arm.

Representative results of camera input and resultant network activity are presented in Fig. (2); as can be seen, visualization of the target increased network activity according to its localization.

Tracking of the target by multiple representative networks is presented in Fig. (3). Visual inspection of these sequences indicates that the robot arm followed the target. Notably, in one of these sequences (network 1), the target was purposely moved out of view of the camera, at which point the robot arm returned to 0 (the midpoint) due to absence of differential signals between the network halves. Tracking resumed when the target was moved back into view (Fig. 3).

Analyses of tracking in these examples demonstrated that the robot arm tracked the target with considerable accuracy; the arm remained within the smallest unit measured ( $<0.25$ ”) for $68 \pm 13 \%$ of the observation period (Fig. 4).

\section{DISCUSSION}

We present herein a model with unique positioning of an ex vivo neuronal network to receive digital environmental input, and to provide responsive output. Since the input corresponds to sensory information, and the output corresponds to motor activity, the ex vivo network functions as a population of interneurons - i.e., a central nervous system that processes incoming information and generates an appropriate response. This unique model can be analyzed in detail, and/or expanded upon to encompass additional functionality. 

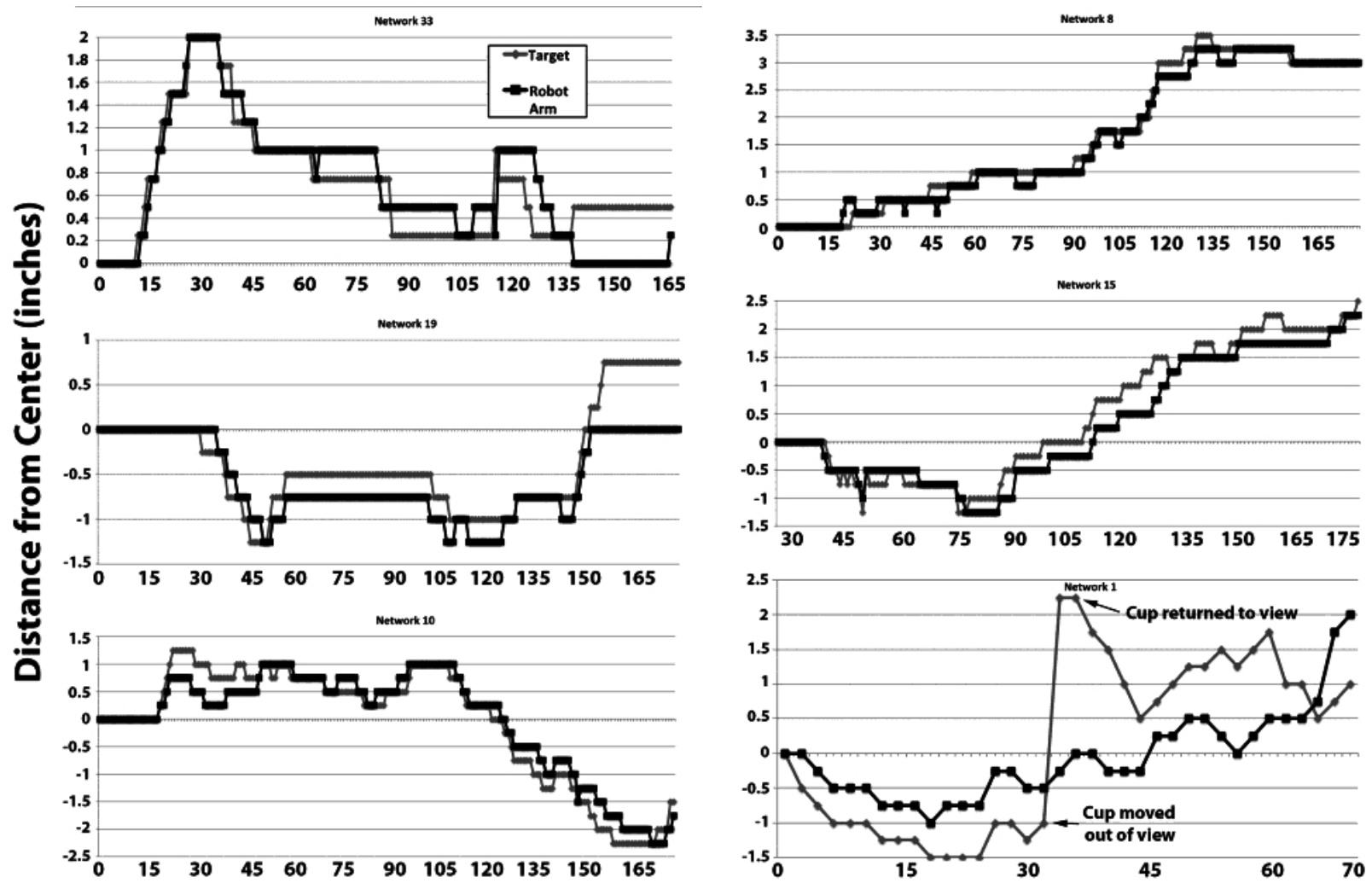

Time (seconds)

Fig. (3). Representative tracking of the target by the robot arm. Mov files of tracking were opened to an identical size, and a ruler was mounted below the on-screen image, with 0 representing the initial localization of the robot arm. Migration of the target and arm were quantified over the indicated time period. Migration was quantified using distance increments of 0.25 ”; + values indicate migration to the right, - values indicate migration to the left.

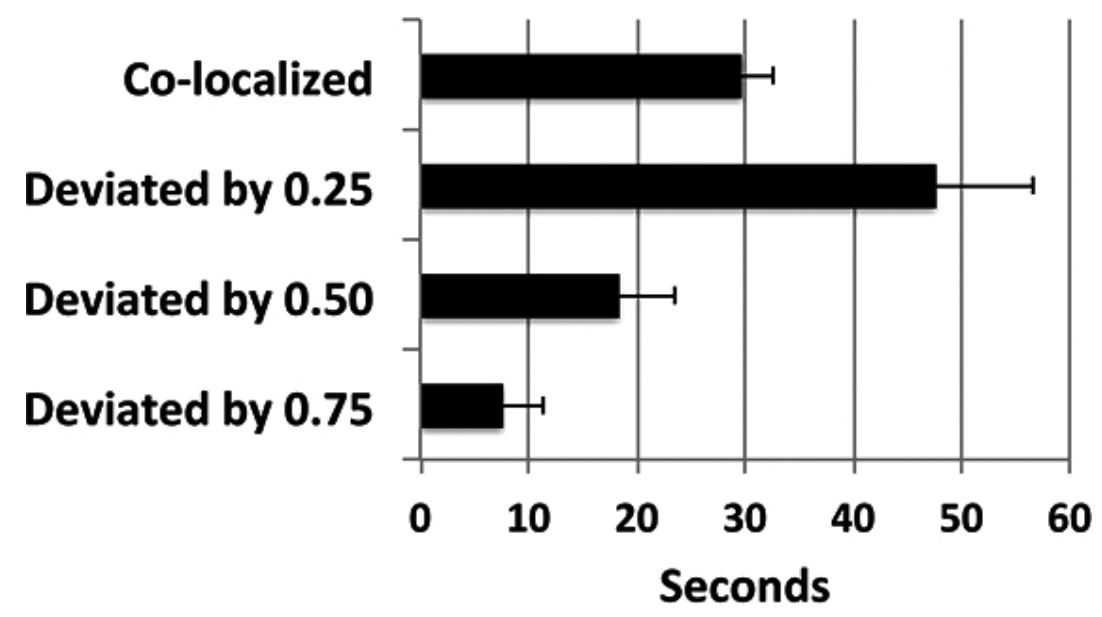

Fig. (4). Average accuracy of tracking. The period of time during which the robot co-localized within the same 0.25 " area as the target, or deviated from the target by various distances, is indicated.

Prior studies demonstrated that these networks display alterations in signaling consistent with long-term potentiation following repetitive stimulation with the same digitized synaptic signal utilized herein $[4,6]$. Embodiment of the neuronal network as described herein provides the unique opportunity for future studies of long-term potentiation by biological neuronal networks in an environmental context. Many studies have resorted to artificial networks, which provide an environment that can readily and precisely be manipulated, but retain the limitation that they are not comprised of actual neurons. Previous studies have utilized similar ex vivo networks to operate a small model vehicle 
within a closed environment and observed network firing patterns in response to physical barriers [25, 26]. The current system expands upon this "reactive" earlier embodiment of neuronal networks in our system monitored active tracking of a target versus passive response.

Setting a threshold for full red pixels allowed us to track the target against a background of mixed coloration that included an additional non-target object that contained red pixels (the human operator). Use of a solid-color background that lacks non-target objects would lessen/eliminate a requirement for thresholding, but would impose an artificial situation that is not reminiscent of real-world dynamics. With the "mixed" background utilized herein, we did not attempt to identify intermediate threshold settings, the minimum target size/shape, degree of additional non-target objects with varying degrees of red pixels, and whether or not the target could contain regions of other hues, all of which would be of interest.

It should be noted that perfect co-localization is not to be anticipated, since the robot responds to net movement of the target; i.e., the target itself must move a distance of $>0.25$ " in either direction to stimulate corresponding movement of the arm. Tracking of the arm could instead be accomplished by digital analyses of target and arm movement. However, this approach involves considerable "stuttering," due to minor differences in pixel recording. Accordingly, we considered the most appropriate method for monitoring tracking was instead to record net movement of the arm within pre-defined increments Fig. (4). An additional compounding factor was that endogenous network activity continued during tracking regimens, which prevented stimulation of the robot arm exclusively by exogenously-derived stimulation.

We have conducted full-range tracking (i.e., full left to full right and back again), as well as maintaining our target exclusively within the left or right regions, with similar tracking in both cases. In one instance in which we removed the target from the field of view, the robot arm returned to the center, and resumed tracking when the target was restored within the field of view (Fig. 4, Network 1).

This is reminiscent of normal brain activity and perception, since synaptic activity persists throughout the brain, over which sensory input and resultant downstream activity is superimposed.

Similarly, optimal tracking was demonstrated by networks that displayed similar levels of spontaneous activity within the left and right domains. Since we converted pixels to our previously-recorded synaptic signal, and did not amplify them above their recorded amplitude, the responsiveness of the robot arm can be diminished should the network display uneven spontaneous activity. In such cases, net movement of the arm favors the side of the network with greater spontaneous activity.

Organotypic (slice) cultures can be established on MEAs [3]. However, networks established from dissociated neurons as used herein can be maintained for several months (and longer under optimal conditions) [25]. Networks generated herein have no architectural restrictions during axonogenesis, which therefore fosters nearest-neighbor connections. Future refinements will include the use of nanocages at plating, to provide a degree of directionality to developing networks. Additional possibilities include establishing of networks from various transgenic mouse models, as well as adding additional neurons to established networks as a model of neurogenesis.

In the particular configuration utilized herein, video input was derived from the digital camera mounted on the Manus robot arm. However, the MEA/computer interface could receive input from any digital camera. "Motor" output could vary from our use of a robot arm to any mechanical/functional activity with an electronic interface.

Future studies could involve a robot-embodied model containing two neuronal networks. The first network, representing the central nervous system, will control tracking of a target via the Manus arm as above. Following successful location of the target, an equivalent number of signals are received by and transmitted from each half of the network, and the arm therefore ceases movement. When no delta in signals between $\mathrm{L}$ and $\mathrm{R}$ hemispheres is detected (e.g. for $\geq 5 \mathrm{sec}$ ), the second network will be activated. Signal streams from the second network, representing the peripheral (motor neuron) system, will then direct the Manus arm to reach forward and retrieve the object; note that the Manus arm has existing programs to support retrieval and object placement. While this represents a simple two-stage embodiment, it parallels our real-world behavior in that humans locate an object of interest via sensory input and motor output (orientation of eyes, head and/or body), and only after localization do we stimulate a second set of motor nerves to initiate object retrieval. Moreover, our recognition of a target occurs over and above a "basal" continuous sensory input, which is analogous to the continuous spontaneous activity of networks during target recognition herein. 


\section{CONCLUSION}

Ultimately, more complex structures than those possible using individual neuronal networks can be developed, allowing exploration of the basic principles about the formation of higher-level neuronal structures. These analyses may contribute to optimization of neuronal-computer interfaces for perceptive and locomotive prosthetic applications. Manipulation of inhibitory neuronal activity and initial connectivity may provide insight into perturbations of balance in excitatory/inhibitory activity that promote seizures and/or perturb motor activity in epilepsy and Parkinson's disease [4 $6,29]$. In addition, the inherent potentially-interfering basal activity of an amplitude matching sensory input in our system may provide a useful system for analyses of conditions such as attention deficit hyperactivity disorder, where competing sensory and internal distractions can distract an individual from task recognition and completion.

Ex vivo neuronal networks as generated herein display rapid inhibition of complex signal patterns in response to sub-cytotoxic concentrations of oligomerized Abeta, and that these alterations are synergistically increased by iron and airborne nanoparticles, yet attenuated by zinc [7 - 9]. Comparison of tracking accuracy of embodied networks prepared from mice harboring key mutations with those from normal mice, accompanied with exposure to Abeta and/or other neurotoxins, may be particularly useful for monitoring subtle impairment of neuronal function.

\section{ETHICS APPROVAL AND CONSENT TO PARTICIPATE}

Not applicable.

\section{HUMAN AND ANIMAL RIGHTS}

No Animals/Humans were used for studies that are base of this research.

\section{CONSENT FOR PUBLICATION}

Not applicable.

\section{CONFLICT OF INTEREST}

The authors declare no conflict of interest, financial or otherwise.

\section{ACKNOWLEDGEMENTS}

This research was supported by Army Research Office grant W911NF-11-1-0125.

\section{REFERENCES}

[1] Hales CM, Rolston JD, Potter SM. How to culture, record and stimulate neuronal networks on micro-electrode arrays (MEAs). J Vis Exp 2010; 39(39): 2056. [PMID: 20517199]

[2] Wagenaar DA, Pine J, Potter SM. Effective parameters for stimulation of dissociated cultures using multi-electrode arrays. J Neurosci Methods 2004; 138(1-2): 27-37.

[http://dx.doi.org/10.1016/j.jneumeth.2004.03.005] [PMID: 15325108]

[3] Zemianek JM, Serra M, Guaraldi M, Shea TB. Stimulation with a low-amplitude, digitized synaptic signal to invoke robust activity within neuronal networks on multielectrode arrays. Biotechniques 2012; 52(3): 177-82. [PMID: 22401551]

[4] Zemianek JM, Lee S, Guaraldi M, Shea TB. Accelerated establishment of mature signaling patterns following stimulation of developing neuronal networks: "learning" versus "plasticity". Int J Dev Neurosci 2012; 30(7): 602-6.

[http://dx.doi.org/10.1016/j.ijdevneu.2012.08.001] [PMID: 22906544]

[5] Zemianek JM, Shultz AM, Lee S, Guaraldi M, Yanco HA, Shea TB. Transient epileptiform signaling during neuronal network development: regulation by external stimulation and bimodal GABAergic activity. Int J Dev Neurosci 2013; 31(2): 131-7. [http://dx.doi.org/10.1016/j.ijdevneu.2012.11.003] [PMID: 23220177]

[6] Zemianek JM, Lee S, Guaraldi M, Shea TB, Shea TB. Critical role for inhibitory neurons in modulation of synaptic signaling in ex vivo neuronal networks. Int J Dev Neurosci 2013; 31(5): 308-10. [http://dx.doi.org/10.1016/j.ijdevneu.2013.03.011] [PMID: 23563174]

[7] Lee S, Zemianek J, Shea TB. Rapid, reversible impairment of synaptic signaling in cultured cortical neurons by exogenously-applied amyloidB. J Alzheimers Dis 2013; 35(2): 395-402.

[PMID: 23403533] 
[8] Lee S, Sohal IS, Thierren M, Pal AK, Bello D, Shea TB. Additive impairment of synaptic signaling in cultured cortical neurons by exogenously-applied oligomerized abeta and airborne nanoparticles generated during Photocopying. J Alz dis 2015; 47 : $49-54$.

[9] Taddeo MA, Lee S, Shea TB. Synergistic inhibition of synaptic signaling in cortical cultures by subcytotoxic levels of oligomerized amyloid$\beta$ and iron: alleviation by zinc. J Alzheimers Dis 2014; 41(2): 365-9. [PMID: 24625798]

[10] Van Pelt J, Corner MA, Wolters PS, Rutten WL, Ramakers GJ. Longterm stability and developmental changes in spontaneous network burst firing patterns in dissociated rat cerebral cortex cell cultures on multielectrode arrays. Neurosci Lett 2004; 361(1-3): 86-9. [http://dx.doi.org/10.1016/j.neulet.2003.12.062] [PMID: 15135900]

[11] van Pelt J, Wolters PS, Corner MA, Rutten WL, Ramakers GJ. Long-term characterization of firing dynamics of spontaneous bursts in cultured neural networks. IEEE Trans Biomed Eng 2004; 51(11): 2051-62. [http://dx.doi.org/10.1109/TBME.2004.827936] [PMID: 15536907]

[12] Madhavan R, Chao ZC, Potter SM. Spontaneous bursts are better indicators of tetanus-induced plasticity than responses to probe stimuli. In: 2nd Int IEEE EMBS Conf Neural Eng.; Arlington, VA. 2005. [http://dx.doi.org/10.1109/CNE.2005.1419652]

[13] Wagenaar DA, Pine J, Potter SM. An extremely rich repertoire of bursting patterns during the development of cortical cultures. BMC Neurosci 2006; 7: 11. [http://dx.doi.org/10.1186/1471-2202-7-11] [PMID: 16464257]

[14] Ikegaya Y, Matsumoto W, Chiou HY, Yuste R, Aaron G. Statistical significance of precisely repeated intracellular synaptic patterns. PLoS One 2008; 3(12): e3983.

[http://dx.doi.org/10.1371/journal.pone.0003983] [PMID: 19096523]

[15] Chiappalone M, Casagrande S, Tedesco M, et al. Opposite changes in glutamatergic and GABAergic transmission underlie the diffuse hyperexcitability of synapsin I-deficient cortical networks. Cereb Cortex 2009; 19(6): 1422-39. [http://dx.doi.org/10.1093/cercor/bhn182] [PMID: 19020204]

[16] Ben-Ari Y. Developing networks play a similar melody. Trends Neurosci 2001; 24(6): 353-60. [http://dx.doi.org/10.1016/S0166-2236(00)01813-0] [PMID: 11356508]

[17] Khazipov R, Khalilov I, Tyzio R, Morozova E, Ben-Ari Y, Holmes GL. Developmental changes in GABAergic actions and seizure susceptibility in the rat hippocampus. Eur J Neurosci 2004; 19(3): 590-600. [http://dx.doi.org/10.1111/j.0953-816X.2003.03152.x] [PMID: 14984409]

[18] Marty S, Wehrlé R, Sotelo C. Neuronal activity and brain-derived neurotrophic factor regulate the density of inhibitory synapses in organotypic slice cultures of postnatal hippocampus. J Neurosci 2000; 20(21): 8087-95. [PMID: 11050130]

[19] Seil FJ. BDNF and NT-4, but not NT-3, promote development of inhibitory synapses in the absence of neuronal activity. Brain Res 1999; 818(2): 561-4. [http://dx.doi.org/10.1016/S0006-8993(98)01304-3] [PMID: 10082848]

[20] Spitzer NC. Electrical activity in early neuronal development. Nature 2006; 444(7120): 707-12. [http://dx.doi.org/10.1038/nature05300] [PMID: 17151658]

[21] Zhang LI, Poo MM. Electrical activity and development of neural circuits. Nat Neurosci 2001; 4(Suppl): 1207-4.

[22] Jimbo Y, Robinson HP, Kawana A. Strengthening of synchronized activity by tetanic stimulation in cortical cultures: Application of planar electrode arrays. IEEE Trans Biomed Eng 1998; 45(11): 1297-304. [http://dx.doi.org/10.1109/10.725326] [PMID: 9805828]

[23] Wagenaar DA, Potter SM. A versatile all-channel stimulator for electrode arrays, with real-time control. J Neural Eng 2004; 1(1): 39-45. [http://dx.doi.org/10.1088/1741-2560/1/1/006] [PMID: 15876621]

[24] Chao ZC, Bakkum DJ, Wagenaar DA, Potter SM. Effects of random external background stimulation on network synaptic stability after tetanization: a modeling study. Neuroinformatics 2005; 3(3): 263-80. [http://dx.doi.org/10.1385/NI:3:3:263] [PMID: 16077162]

[25] Demarse TB, Wagenaar DA, Blau AW, Potter SM. The Neurally Controlled Animat: Biological Brains Acting with Simulated Bodies. Auton Robots 2001; 11(3): 305-10. [http://dx.doi.org/10.1023/A:1012407611130] [PMID: 18584059]

[26] Novellino A, D’Angelo P, Cozzi L, Chiappalone M, Sanguineti V, Martinoia S. Connecting Neurons to a Mobile Robot: An In Vitro Bidirectional Neural Interface. Compute. Intelligence Neurosci. 2007.

[27] Serra M, Guaraldi M, Shea TB. Inhibitory neurons modulate spontaneous signaling in cultured cortical neurons: density-dependent regulation of excitatory neuronal signaling. Phys Biol 2010; 7(2): 026009. [http://dx.doi.org/10.1088/1478-3975/7/2/026009] [PMID: 20526031]

[28] Mahmud M, Bertoldo A, Girardi S, Maschietto M, Vassanelli S. SigMate: A MATLAB-based neuronal signal processing tool. Conf Proc IEEE Eng Med Biol Soc 2010; 2010: 1352-5.

[PMID: 21096329] 
[29] Therrien M, Vohnoutka R, Boumil E, Guaraldi M, Lee S, Shea TB. Insufficient developmental excitatory neuronal activity fails to foster establishment of normal levels of inhibitory neuronal activity. Int J Dev Neurosci 2016; 55: 66-71.

[http://dx.doi.org/10.1016/j.ijdevneu.2016.09.009] [PMID: 27686511]

\section{(C) 2017 Shultz et al.}

This is an open access article distributed under the terms of the Creative Commons Attribution 4.0 International Public License (CC-BY 4.0), a copy of which is available at: https://creativecommons.org/licenses/by/4.0/legalcode. This license permits unrestricted use, distribution, and reproduction in any medium, provided the original author and source are credited. 